\title{
Focus socio-psychologique sur la crise politique belge de 2011
}

\author{
Entretien croisé avec Valérie Rosoux et René Zayan
}

\author{
Stéphane Baele, Valérie Rosoux, René Zayan
}

\begin{abstract}
Résumé/Absract
[Français] Dans ces pages, nous plaçons la situation politique belge actuelle sous la lumière de l'analyse socio-psychologique. Répondant aux questions de Stéphane Baele (Université de Namur), le Professeur René Zayan (Université catholique de Louvain) et la Professeur Valérie Rosoux (Université catholique de Louvain), tous deux experts en psychologie sociale et politique, donnent ici en chassé-croisé une lecture décalée de la crise de formation gouvernementale qu'a connue la Belgique en 2007-2011. Leurs approches, différentes mais complémentaires, mettent en avant des éléments comme le charisme, le traumatisme, ou les représentations mentales.

Mots-clefs: psychologie, politique, Belgique, conflit, charisme, négociations, Belgique, Zayan, Rosoux, crise politique belge (2007-2011).

[English] In the following pages, the political crisis that Belgium experienced in 2007-2011 is put under a socio-psychological light, thanks to a discussion led by Stéphane Baele (University of Namur) with two leading experts in political psychology and philosophy and acute observers of the Belgian political scene. Even if their methods and theoretical assumptions differ, Prof. Rosoux and Prof. Zayan (both at Université catholique de Louvain) offer together a fresh, complex, original frame of understanding of Belgian record-breaking governmental negotiations.

Keywords: Psychology, Belgium, politics, conflict, charisma, negotiations, Zayan, Rosoux, Belgian political crisis (2007-2011).

Stéphane Baele : C'est une habitude des contributeurs de notre revue : ils préfèrent chercher les réponses à leurs questions là où le lecteur les attend le moins. En l'occurrence, nous aimerions ici, pour éclairer la crise politique belge, nous détacher du cadre des analyses politiques classiques pour laisser la place à un effort de compréhension de type psychologique ou philosophique. Prof. René Zayan, vous enseignez l'éthologie et la psychologie politique à l'Université Catholique de Louvain et par ailleurs vous avez signé une série d'interventions médiatiques originales et percutantes visant à expliquer les résultats d'élections (notamment les
\end{abstract}


scrutins français, avec votre documentaire Coupez le son). Prof. Valérie Rosoux, vous êtes philosophe et politologue et enseignez, vous aussi, à l'Université catholique de Louvain. Vos recherches portent principalement sur le rôle et l'utilisation politique de la mémoire collective, des représentations sociales du passé. Notre première question balaie large et vous est adressée à tous les deux : quelle est votre lecture de la crise politique belge? Est-elle différente, complémentaire, compatible avec les grilles d'analyse habituelles des politologues?

Valérie Rosoux : Mon angle d'approche est à certains égards paradoxal. Il est en effet lointain et proche. Lointain, au sens que la politique intérieure belge n'a jamais constitué un cas d'étude en tant que tel dans mes recherches. J'ai consacré ma thèse aux représentations du passé dans la politique étrangère de la France à l'égard de l'Allemagne et de l'Algérie. Je me suis ensuite tournée vers le poids du passé dans un contexte post-conflit, depuis les Balkans jusqu'au Rwanda. La Belgique peut donc sembler bien lointaine de mes préoccupations. Elle n'en demeure pas moins proche à au moins deux égards. Sur le plan du vécu d'abord. Je pense ici au quotidien d'un quartier peuplé principalement d'immigrés et de jeunes flamands particulièrement dynamiques, ou encore à l'expérience journalière d'un enseignement néerlandophone accueillant des enfants principalement francophones. Au-delà du vécu, je pense également au processus de négociation qui se donne à voir au jour le jour. J'enseigne la négociation et le cas belge, même s'il ne figure pas parmi les cas dont je suis spécialiste, est emblématique à maints égards. Il apparaît comme un cas d'école tant les réactions des uns et des autres sont contrastées, tant les impasses sont répétées. L'adjectif « surréaliste » ne me paraît pas exagéré au vu de certains blocages.

J'ai envie d'ajouter que c'est au cours de mon séjour aux États-Unis, donc relativement loin, que la Belgique est devenue plus centrale comme objet d'étude en tant que tel. C'est suite aux questions de mes collègues au United States Institute of Peace (USIP) que j'ai réfléchi aux variables qui permettaient de comprendre une telle crispation, une telle sensibilité de chaque côté de la frontière linguistique. Mon questionnement pourrait être résumé comme suit : comment concevoir que la charge émotionnelle de certains dossiers puisse empêcher toute forme de pragmatisme ? Cette question renvoie selon moi à des nœuds mémoriels, encore relativement peu explorés. L'article consacré aux traumatismes dans ce numéro est une belle exception en la matière.

Dernier point, mes propos montrent que je m'inscris dans une perspective épistémologique particulière. Dans chacune de mes recherches, j'essaie de prendre en considération la manière dont les acteurs politiques mettent en scène le passé. Or ces mises en scène ne sont jamais des descriptions rationnelles, complètes et scientifiques, mais des représentations particulières. En ce sens, il me semble que l'analyse ne peut restreindre son exploration au seul domaine de la pensée organisée, 
rationnellement construite et logiquement conduite. Elle doit compléter le modèle de la décision rationnelle par la prise en compte des perceptions et des interprétations des acteurs. Il importe, en d'autres mots, de garder à l'esprit que ce ne sont pas seulement les événements eux-mêmes, mais aussi - et surtout - la représentation de ceux-ci qui influencent la prise de décision. L'un des exemples les plus révélateurs en la matière concerne la légende des $80 \%$ de soldats flamands morts dans les tranchées durant la Première Guerre mondiale pour une cause qui n'était pas la leur. Cette représentation est clairement démentie par les historiens (Flamands et Francophones). Elle n'en demeure pas moins l'expression réelle d'une humiliation dont il faut tenir compte pour comprendre un tant soit peu la nature du débat belgobelge.

René Zayan: L'approche d'un psychologue, l'approche d'un spécialiste de la communication politique, devrait en principe différer de celle des politologues. On constate que les politologues qui ont été interrogés quasiment tous les jours durant cette crise-là sont ce qu'on peut appeler, d'une manière générale, des commentateurs $\mathrm{au}$ jour le jour des événements ou des non-événements qui ont eu lieu; ils envisagent, quand on leur demande, des prédictions sur ce qui risque d'arriver (par exemple, savoir si Mr De Wever continue ou pas dans la même ligne de revendications radicales). Le psychologue va s'intéresser à ce qui est en jeu. Premièrement, dans les représentations mentales que les acteurs de la négociation produisent à propos de leur partenaire ; deuxièmement, dans la manière dont ils vont exprimer verbalement ou non-verbalement (c'est-à-dire par le visage, par la voix, par les postures) l'état de leurs négociations.

J'aimerais rappeler - vous pouvez en trouver trace - que j'ai dit au lendemain de l'élection que Bart De Wever fera tout pour ne pas appartenir à un gouvernement fédéral. Comment se fait-il qu'un Marseillais puisse prédire cela, alors que d'autres ont pu croire l'inverse ? Il a suffi de tirer les conclusions de sa démission du tandem formé avec la CD\&V à la suite des élections de 2007, laissant MR. Leterme diriger. Je me suis toujours intéressé à l'émergence des mouvements nationalistes européens, particulièrement aux mouvements de type régionaliste et identitaire de type «Flandre », aux mouvements qui ont été créés en 1933-1934. Je savais dès lors que $\mathrm{Mr}$ De Wever était un républicain (donc antimonarchiste), qu'il était un nationaliste (de son propre aveu), et du point de vue économique qu'il se situait clairement du côté du libéralisme. Donc, les choses sont très claires : il a eu une stratégie d'aller-retour, de création de frustrations et de faux espoirs, menant tout le monde en bateau jusqu'au moment où les Francophones se sont enfin rendus compte combien ses revendications étaient loin de ce qu'ils avaient pu imaginer, eux qui avaient vécu pendant des années dans ce qu'on peut appeler le «mythe de la Belgique unie » tandis que depuis longtemps de nombreux Flamands disaient «ça 
ne peut plus continuer comme ça », revendiquant pour le moins une autonomie. On savait cela, c'était très clair, tout était prévisible.

Mettez-vous à la place d'un politicien, devant Mr De Wever qui a une telle stratégie de négociation ; comment «dealer » avec cela quand on est francophone ? Voilà un problème de psychologue. Quand, en plus, on ne maitrise pas spécialement le néerlandais (ce qui n'est pas le cas de quelqu'un comme Didier Reynders, mais il n'était pas à la table des négociations durant un an), comment faire ? Il s'agissait avant tout de voir que Mr De Wever était dans une logique de conflit. Pour lui la politique ce n'est pas comme pour les francophones une vieille tradition de conciliation, de pacification, et de compromis. Sa définition du politique est issue de Carl Schmitt - et donc de Nietzsche -: la politique est la désignation des amis et des ennemis. Pour Bart De Wever, mieux vaut donc avoir de vrais ennemis que de faux amis. Voilà donc un problème psychologique. Bart De Wever allait nécessairement commencer par être souple, pour donner des espoirs, et ensuite se rétracter. Et pourtant rappelez-vous toutes ces interviews de négociateurs s'étonnant de ne pas arriver à boucler le communautaire. La population reçoit alors l'image d'un Bart De Wever radical mais dominant, image renforcée par cette technique très intéressante de faire soudain marche arrière pour mieux se faire rappeler.

Stéphane Baele : Cette tactique d'aller-retour a contribué à entrainer, c'est un fait attesté par un record, une très longue période de négociation et de formation gouvernementale. À de nombreux égards, cette période a pris les atours d'un feuilleton, d'une pièce de théâtre au scénario tissé de temps de stagnation et de moments de dramatisation; pour certains négociateurs cette situation fut également dramatique sur le plan personnel (naissances et décès de proches). Professeur Rosoux, quels épisodes de ce film vous ont particulièrement frappés ? Quels acteurs vous ont paru être les meilleurs (ou les pires) et pour quelle raison?

Valérie Rosoux: Travaillant le plus souvent à l'étranger, je n'ai pas suivi au jour le jour les épisodes de ce «feuilleton», pour reprendre votre expression. Lors de mon séjour à Washington, j'ai pris l'habitude de dialoguer à ce sujet avec un collègue flamand (nous étions les seuls Belges travaillant au sein du US Institute of Peace qui regroupe une centaine de chercheurs venus du monde entier). Dès notre première rencontre, nous avons décidé de ne pas éviter le «sujet Belgique ». Et petit à petit, nous avons mis en place une forme de concertation permanente des plus enrichissantes. Le principe était simple. Il s'agissait de discuter des événements à partir de deux couvertures médiatiques différentes. Jonas Claes prenait connaissance de la presse néerlandophone alors que je me concentrais sur la presse francophone. Il n'était pas question de scruter les événements en tant que tels. Notre démarche n'avait rien d'une enquête d'ordre historiographique. Il s'agissait davantage de discuter de nos perceptions, de nos inquiétudes éventuelles, de nos étonnements, de 
nos incompréhensions. De tous ces aller-retour, je retiens essentiellement le caractère non seulement pluriel des représentations (phénomène en soi normal et même plutôt sain au sein d'un État regroupant des communautés différentes), mais aussi le caractère contradictoire de nombre de ces représentations. Les points de vue n'étaient pas seulement divergents, ils étaient souvent incompatibles. Dans un tel cas de figure, il est extrêmement difficile de pouvoir aborder la négociation de manière constructive et intégrative. La vision d'un jeu à somme nulle s'impose. L'objectif est dès lors de défendre bec et ongles ses acquis et son territoire, au sens strict et symbolique. Le manque de confiance est tel qu'il empêche toute souplesse et toute créativité.

Quant à la question des acteurs en tant que tels, je ne souhaite donner ni bons points, ni mauvais points. Il ne s'agit pas pour moi d'évaluer quiconque. Quelle serait d'ailleurs ma légitimité en la matière ? Je suis davantage interpellée par des comportements, par des silences aussi, j'ai presque envie de dire par des « fantômes ». En cela, les acteurs qui me frappent le plus ne sont peut-être pas ceux que nous voyons et entendons, jour après jour, au fil des négociations marathons qui se succèdent. Ce sont les fantômes, je veux dire par là ceux qui, bien qu'absents de la scène politique actuelle semblent peser de tout leur poids. J'ai parfois l'impression qu'il y a bien plus d'invités à la table de négociations qu'il n'y parait à première vue. Je ne cible pas ici tel ou tel personnage politique précis. D'autres observateurs, plus experts que moi en politique intérieure belge, peuvent se risquer à l'exercice. Je pense davantage au poids de certaines figures familiales, au poids de l'humiliation qui n'en finit pas de se faire sentir entre les lignes des communiqués. $\mathrm{Au}$ poids du ressentiment non clairement exprimé et sans doute profondément ancré dans nombre de foyers belges. La récurrence et l'intensité du débat sur l'amnistie le soulignent à l'envi. Comment comprendre cette crispation sans se référer au poids de ceux que l'on essaie de réhabiliter d'une part et de ceux qu'il s'agit d'honorer et de ne pas oublier de l'autre ? Cette question ne concerne pas exclusivement à l'héritage de la Seconde Guerre mondiale. Nous avons réfléchi à la question, l'historienne Laurence van Ypersele et moi-même, dans un article récent. La Première Guerre mondiale est, selon nous, l'origine d'une pluralité de mémoires sans précédent dans l'histoire du pays. Elle représente en quelque sorte la matrice d'une mémoire conflictuelle.

Stéphane Baele : Il est vrai que ce conflit est vecteur de plusieurs couples traumatismes/rituels - pour reprendre la typologie que je développe dans ma contribution au présent volume («Traumatismes et rituels du nationalisme flamand ») - ancrés dans la mémoire collective flamande. Les récents débats et polémiques autour des commémorations à venir pour célébrer le centenaire du conflit en témoignent. Au vu de ces lectures contradictoires du passé, chaque négociation est, comme vous l'avez dit Prof. Rosoux, une occasion de tension. Et, à 
cet égard, on est en droit de se demander si le calendrier politique à venir n'est pas propice à une exacerbation des tensions. Car si l'État est peut-être sorti de cette crise de formation gouvernementale, d'autres élections se préparent, des échéances de réformes institutionnelles sont demandées, de multiples «plans B » sont évoqués comme si le langage n'avait pas d'effet autre qu'électoral. Une question émerge à cet égard pour vous, Prof. Zayan. Au vu de ce que vous avez observé en termes de charisme, de comportements, quelles personnalités ont été marquantes et sont susceptibles d'émerger/de s'effondrer à l'occasion des prochains scrutins?

René Zayan: En ce qui concerne le charisme en tant que tel, la tradition démocratique parlementaire belge - surtout francophone - exclut une expressivité très charismatique. La Belgique est un pays où du point de vue du comportement non-verbal les leaders sont modestes, assez neutres, sont conciliants, certainement pas dominants comme les Français par exemple, certainement pas arrogants. Leur débit de parole est lent, ils n'interrompent pas, ils ne veulent pas dominer par la parole. Ils sont, en somme, accommodants et démocratiques dans leurs comportements. Personne n'est vraiment charismatique.

Néanmoins, certains éléments du charisme universel se retrouvent typiquement en Belgique. En ce sens, et comme je le dis dans mon documentaire qui sort sous peu, Elio Di Rupo était la personne qui était la plus à même d'être Premier ministre pour deux raisons. La première est qu'il était socialiste et qu'il a joué des engagements socialistes - à savoir ne pas appauvrir davantage les pauvres, maintenir une solidarité sociale, lutter contre le capitalisme financier -, expliquant à ses nombreux électeurs qu'il était mieux que ce soit un socialiste qui dirige le pays. Du point de vue purement politique, il y avait une adéquation : il était «at the right time at the right place». La seconde raison est plus psychologique. Monsieur Di Rupo a une grande qualité : il est empathique. C'est un monsieur qui n'est jamais arrogant, jamais dominant (au sens strict), il ne montre pas souvent qu'il est assuré, se laissant voir déstabilisé dans les débats. C'est quelqu'un qui est à l'écoute des gens, c'est-àdire que quand on pose une question, il écoute vraiment et ne répond pas automatiquement; il se met à la place des autres. Il a la qualité empathique de montrer, par son comportement, par son regard, par ses gestes, qu'il a compris la question posée, qu'il veut y répondre. En outre, depuis qu'il a mené les négociations, on remarque une nouvelle qualité de l'empathie, associée au leadership : «je comprends que vous avez des problèmes, nous tous ensemble avons des problèmes, je suis proche de vous et non pas d'un autre monde, donc je jouis de la représentativité politique. Je travaille dur pour vous, et avec le gouvernement je peux vous aider, car vous avez besoin d'être aidés ». C'est là une attitude à la fois empathique mais aussi dominante.

Une autre personnalité tout à fait remarquable à cet égard - mais les gens oublient qu'il y avait un gouvernement faisant fonction -, et qui jouit d'une autorité 
internationale considérable en matière socio-économique est Didier Reynders, qui a su garder la main sur une mécanique technique de premier niveau. Mme Onkelinx, avec sa routine de travail, avec son expérience, est également à mettre en avant comme femme d'État, tout comme Mme Milquet. Monsieur Leterme, dans sa situation particulière, a réussi à faire bonne figure. Monsieur De Wever, dans sa logique de ne pas participer à un gouvernement fédéral, vise encore à séduire et récupérer les autonomistes des partis traditionnels flamands, et cette stratégie passera ou cassera. Elle passera dans la mesure où la politique actuelle est en échec ou plutôt dans la mesure où Bart De Wever parvient à faire croire qu'il y a un échec. Dans ce dernier cas, les élections prochaines verront 40 à $42 \%$ de votes flamands aller à la N-VA.

Stéphane Baele : Professeur Zayan, lorsque l'on vous lit ici et quand on vous écoute à l'occasion de vos interventions médiatiques (et de vos enseignements), on est frappé d'entendre que le contenu des discours des hommes et femmes politiques ne pèse que très peu dans leur succès ou échec - en contraste avec la forme, la mise en scène de ces discours. Pensez-vous qu'un tel point de vue soit compatible avec les résultats de la sociologie électorale classique, qui s'attache à expliquer le vote donc le succès et l'échec des mandataires - par la mise en avant de particularités sociologiques (vote des femmes, des étrangers, des jeunes, des classes socioéconomiques, etc.), voire par le recours aux théories du choix rationnel ?

René Zayan : Il faut d'abord rappeler une chose sur ma méthode d'analyse politique: mon approche du politique par le charisme est, contrairement à ce qu'on peut croire, très originale. L'approche du leadership, des catégories de comportement universelles ou culturelles permettant aux votants de se faire une opinion en deux ou trois secondes, est novatrice par rapport aux méthodes politologiques classiques. Des fois, des prévisions correctes du résultat d'élections peuvent être effectuées sur base de photographies vues quelques millisecondes - c'est extraordinaire, absolument hallucinant ! J'ai établi un score de charisme, dont l'échelle va de -100 à +100 , et je peux vous dire que quand un politicien est proche de zéro c'est déjà pas mal, car je vous l'ai dit la majeure partie des politiciens ici sont en fait anti-charismatiques. Sur cette échelle, Ronald Reagan a +90 , Jacques Chirac +82 , et Nicolas Sarkozy seulement +17 en 2007, tandis que Barack Obama avait +22 , et Didier Reynders +92. A l'opposé, François Bayrou score -98, et de nombreux politiciens belges ont un score semblable. Tout cela est prédictif; je peux vous prédire à $85 \%$, à partir de dix secondes de vidéo sans le son d'un débat électoral, qui gagnera. Avec le son, les observateurs habituels prédisent à un taux d'environ $50 \%$ - soit le hasard... Il ne faut donc pas écouter les candidats, car cela brouille l'attention que vous devez porter aux mimiques faciales, aux comportements, qui sont universellement reconnaissables en moins d'une seconde. Pour énoncer une proposition complète à 
la télévision, ces derniers mettent dix à trente secondes, et souvent ils parlent pour ne rien dire. Le cerveau remarque, par tranche de 300 à 500 millisecondes, des choses telles que les clignements des yeux, la voix qui tremble, les sourcils qui se relèvent, et donc en moins de six secondes le spectateur s'est déjà fait une représentation consensuelle des candidats, lui prêtant une série de traits: est-il assuré, est-il intelligent, est-il compréhensible ? Aux leaders charismatiques les spectateurs attribuent de l'intelligence sociale, de la gentillesse, du sens de la responsabilité, etc. Bref, toutes les qualités politiques pour lesquelles on vote. Donc le non-verbal pur est prédictif des élections, et le non-verbal avec le contenu ne l'est plus. Plus encore, se baser sur du texte écrit enlève $60 \%$ de l'information. Même à partir du son complet, c'est beaucoup plus la voix seule que ce qui est dit qui a un impact.

Cela étant dit, l'approche sociologique, l'approche sur les préjugés, sur les contextes, fait partie des sciences politiques; ces ingrédients-là donnent ce qu'on appelle la «political psychology». On pose alors des questions comme : quelles représentations mentales vont faire qu'on est conscient ou non de nos préférences politiques (par exemple démocrate ou républicain américain)? Quels sont les mécanismes qui font que je trouve qu'un candidat correspond à ces représentations mentales, à ces préférences sur ce que constituent les valeurs de base des républicains/démocrates ? Comment expliquer certaines dissonances à cet égard (pour prendre l'exemple supra, Ronald Reagan plaisait à de nombreux démocrates, mais aussi aux républicains - alors qu'il prônait une couverture médicale généreuse) ? Tous ces éléments sont intéressants, et complémentaires à mon approche, que je qualifie donc d'approche comportementaliste du charisme et du leadership.

Ceci étant dit, il s'agit de ne pas confondre information et communication. L'information est un contenu de messages et de concepts qui, par rapport à un état précédent de relative ignorance, apporte une quantité de connaissances significative. $\mathrm{Si}$ vous dites ce que tout le monde sait, vous n'apportez aucune information. Il faut donc venir avec des méthodes novatrices.

En somme, la synthèse est très simple: si en tant que politicien votre communication est géniale (charismatique), votre information sera positivement perçue, qu'elle soit bonne, neutre ou mauvaise. Si votre communication est mauvaise (si vous n'êtes pas charismatique), votre information sera perçue négativement, même si votre texte est excellent. Ce que j'ai toujours voulu, c'est donc que les leaders de partis démocrates traditionnels soient porteurs de charisme et de leadership, afin de faire concurrence aux leaders populistes charismatiques, qui peuvent dire n'importe quoi et susciter l'adhésion.

Stéphane Baele: Il nous semble utile de se pencher sur ce «n'importe quoi », car le contenu des discours est quand même rarement innocent. On remarque ainsi que, 
plus que tout autre responsable politique belge, Mr. De Wever a recours dans son discours à des «mythes » politiques, c'est-à-dire met en avant une série d'événements du passé pour appuyer les axes forts de son programme et encourager parmi l'électorat flamand l'appartenance à une identité politique et communautaire commune. Cette utilisation politique du passé entre en plein dans vos recherches, Prof. Rosoux. Pourriez-vous préciser votre pensée à cet égard ?

Valérie Rosoux : Je voudrais ici prendre un peu de hauteur par rapport au cas belge en insistant sur une distinction qui me paraît éclairante. Je songe ici à la tension qui s'établit entre d'une part le choix du passé et d'autre part le poids du passé. La première perspective montre que toute politique détermine une certaine interprétation de l'histoire. On peut parler à cet égard de reconstruction du passé. La seconde rappelle que la politique est à son tour façonnée par l'histoire. Il ne s'agit plus de la reconstruction du passé, mais de ses empreintes. Quelques mots seulement pour illustrer ces deux dynamiques, en constante interaction dans la pratique, mais néanmoins utiles à distinguer pour contribuer à la compréhension des processus en jeu, par exemple en Belgique.

Considérons un instant le choix du passé. À la différence des mémoires artificielles, la mémoire humaine ne se réfère pas au passé de manière neutre et objective. Ce ne sont pas les choses elles-mêmes qui entrent dans la mémoire, mais leurs représentations. La mémoire ne peut être le reflet exact et parfait du passé. Elle n'en est que la trace. C'est en ce sens que Saint Augustin définit la mémoire comme le «présent du passé ». Force est de constater que les souvenirs ne sont pas littéralement conservés, mais plutôt reconstruits, remaniés en fonction des circonstances. L'attitude du général de Gaulle à l'égard du passé franco-allemand le montre à satiété. Loin d'être constante, elle varie d'une période à l'autre. Entre la Première et la Seconde Guerre mondiale, Mr. de Gaulle décrit l'hostilité naturelle, l'incompatibilité ontologique et la méfiance quasi viscérale qui existent entre Français et Allemands. Quelques années plus tard, quand le rapprochement francoallemand apparaît comme une nécessité pour la France, le même Mr. de Gaulle souligne la complémentarité tout aussi naturelle des deux peuples et les affinités profondes qui les ont toujours attirés. Ce type de réinterprétation du passé n'est concevable que si l'on remet en question le préjugé tenace, selon lequel seul le futur serait ouvert et indéterminé, le passé étant fermé et déterminé. Le passé n’est en réalité jamais pleinement révolu. Bien sûr, les faits passés sont ineffaçables. Nul ne peut défaire ce qui a été fait ou faire que ce qui est advenu ne se soit pas produit. Mais, comme le montre bien Paul Ricœur, le sens de ce qui est arrivé n'est jamais fixé une fois pour toutes. Le caractère sélectif et fluctuant de la mémoire n'est pas un attribut négatif, mais fonctionnel - ou inhérent - de tout recours au passé. En Belgique, la narration officielle du passé, qu'il s'agisse de la bataille des éperons d'or épinglée dans les milieux nationalistes flamands ou de l'indépendance de la 
Belgique racontée par Henri Pirenne, repose tout entière sur ce mécanisme d'ajustement du passé au présent. Inspirée par un intérêt actuel, elle tend à une fin actuelle. Le passé ne se réduit pourtant pas à un réservoir d'instruments que l'on sélectionnerait au gré des intérêts et des objectifs. Il constitue également une contrainte à laquelle peu d'acteurs politiques échappent. C'est ici qu'il importe de garder à l'esprit le poids du passé. La force de certains souvenirs personnels et familiaux le rappelle souvent clairement.

Cette distinction permet d'éclairer la question des « mythes » politiques. A priori, les événements présentés comme fondateurs pour l'identité politique flamande semblent relever des usages du passé. Mais ici se pose la question de savoir dans quelle mesure certaines représentations et plus précisément certaines déformations du passé sont faites de manière consciente ou non. L'homme politique est-il libre et donc responsable - lorsqu'il élabore sa lecture de l'histoire, ou bien est-il luimême l'objet d'un prisme cognitif qui biaise son interprétation historique ? Il me paraît extrêmement difficile d'identifier avec certitude l'intentionnalité des acteurs. L'étude de certains cas jugés extrêmes, comme celui des Balkans, permet d'émettre des hypothèses en la matière. Je songe ici à l'attitude adoptée par un leader tel que Slobodan Milosevic. Il est effectivement frappant que la montée au pouvoir de ce dernier corresponde précisément au moment de la relance des arguments historiques et des revendications qui en découlent. C'est bien en réveillant des peurs traditionnelles que Slobodan Milosevic assure son ascension. C'est en faisant bouillir les sentiments d'insécurité et de fierté de ses compatriotes serbes du Kosovo qu'il finit par s'imposer à la direction collégiale du pays. Il apparaît en tout cas que Mr. Milosevic édifie progressivement son pouvoir en récupérant la lutte nationaliste que le discours communiste cherchait à apaiser. En lieu et place de l'égalité et la fraternité, il semble exhumer les oripeaux du passé qui sèment la haine et le chauvinisme. Dans ce cas précis, la volonté de ne retenir que les traits douloureux du passé semble bien réelle quand on songe aux exagérations, aux silences, aux généralisations, aux inexactitudes qui ont caractérisé le discours officiel de Belgrade pendant plus de dix ans.

Je cite cet exemple car il est un cas d'école à maints égards. Qu'en est-il pour la Belgique ? Nous ne sommes pas dans un contexte de guerre civile, de violence physique. Mais il ne s'agit pas non plus d'un débat apaisé au sujet du passé dit « national », pour peu que cette notion ait encore du sens en Belgique. La controverse liée à la question de l'amnistie montre à quel point ce passé demeure problématique. D'où l'intérêt de prendre cette dimension au sérieux.

Stéphane Baele: Il me semble que c'est ici que se rejoignent vos deux champs de recherche, Prof. Zayan et Prof. Rosoux : on constate que souvent, les personnalités politiques qui s'inscrivent fortement dans cette logique de «choix du passé », de réécriture simpliste de l'histoire, sont capables de convaincre, de mobiliser 
l'opinion, de réaliser, performativement, des modifications identitaires chez leurs auditeurs. En d'autres mots, ils sont souvent charismatiques. Parfois, ils sont taxés de populisme, mais est-ce bien la même chose ? Ainsi, Prof. Zayan, de nombreux politologues attestent de la réussite de la N-VA comme de celle d'un parti populiste (il faut cependant noter que cette dénomination ne fait pas l'unanimité), en mettant en avant la présence d'un leader fort et charismatique, le présence dans le discours de thématiques électoralement porteuses, la présentation simpliste de ces thématiques (à l'instar de la mise en scène des camions remplis de billets partant de Flandre et arrivant en Wallonie), etc. Cette notion de populisme est-elle pertinente, est-elle compatible avec le genre d'analyses que vous menez, avec votre injonction de «couper le son» pour mieux observer les comportements? Comment comprendre le trio populisme/charisme/nationalisme?

René Zayan: L'histoire montre effectivement que, malheureusement, les leaders populistes sont plus charismatiques que les autres; ils manifestent plus d'expressivité, de dominance, d'empathie. L'empathie en particulier est très rare chez les politiciens classiques, à qui l'on reproche de vivre dans une tour d'ivoire, tandis que les leaders populistes sont avec le peuple, vivent comme lui, se comportent comme lui, ont les mêmes représentations mentales, les mêmes désirs. De ce point de vue-là, on peut dire que beaucoup de politiciens traditionnels sont extrêmement inhibés, discrets, et misent beaucoup sur la parole. À la télévision et dans les meetings retranscrits, le non-verbal compte beaucoup plus que la signification des textes - sauf si ce sont de très bons textes (avec des mots-clefs émotionnels, une logique facile et implacable).

Sachant cela, on peut dire que Filip de Winter (leader du Vlaams Belang) est une personnalité populiste charismatique. Mais ce n'est pas le cas de Bart De Wever, qui reste extrêmement calme, assuré, neutre, mais qui n'est pas particulièrement jovial, pas particulièrement social, pas particulièrement empathique, et pas non plus particulièrement dominant. Il est par contre comme beaucoup de gens sont. Pour un Marseillais comme moi, il apparait directement que la communication politique des mandataires au Nord du pays est différente de celle de ceux du Sud. Il s'agit en définitive de parler du populisme d'une manière non ambigüe mais complexe. J'ai à cet égard toujours mis en garde sur le fait que l'Europe génère des mouvements politiques identitaires dont certains sont ethnicistes (pas tous) et nationalistes certains régionaux, certains d'un « empire » européen. De ces mouvements, il y en a deux formes. La première, personnalisée par quelqu'un comme Jorg Haider, est maligne, c'est le populisme autoritaire et xénophobe. Force est de constater que l'Europe est faible et ridicule face à ces mouvements, parce qu'elle n'apprend pas à les connaître. En 2000 déjà, je mettais en garde vis-à-vis de Voktor Orban - vous avez vu ce qu'il fait aujourd'hui. Mais à côté de cela, il y a divers autres populismes qui ne sont pas nécessairement nationalistes, ni ethnicistes, et qui se veulent 
simplement représentatifs du peuple. Il s'agit là d'un populisme démocratique, et peut s'agir d'un populisme qui a émergé, historiquement, sur des démocraties décadentes, comme en février 1934. Depuis le Général Boulanger, ce populisme-là a permis à la démocratie qu'elle devait se régénérer, se repenser. D'une certaine manière, un tel populisme a été bénéfique à la démocratie. En ce sens, et j'ai été le premier à dire voici quelques années, que la notion de populisme ne doit ainsi pas être vue comme étant a priori méprisable. Confondre populisme et fascisme ou confondre, comme c'est le cas ici, populisme et nationalisme xénophobe flamand, ça n'est pas correct. L'un n'implique pas nécessairement l'autre. Charles de Gaulle, François Mitterrand étaient populistes. Ronald Reagan, probablement le plus grand président américain avec Roosevelt, l'était aussi, il a même obtenu tous les votes de confiance au Sénat pourtant majoritairement démocrate ; il laissait croire qu'il était inculte, qu'il était bête, il se moquait de lui-même. C'était un grand président populaire. Jacques Chirac, lui aussi, a été élu comme populiste en 1995. Élu par les hommes, par les femmes, parce qu'il touche le cul des vaches, parce qu'il a fait 500.000 interventions personnelles sur sa carrière - c'est ça le populisme, ce n'est pas nécessairement méchant. À titre personnel je me méfie beaucoup plus, à l'inverse, de quelqu'un comme Mr. Sarkozy.

Stéphane Baele: Votre franc-parler amène, il me semble, de nombreux enseignements qu'un discours trop feutré manquera de faire ressortir. Mais une telle stratégie s'avère parfois dangereuse. On se souviendra notamment de la polémique qui avait opposé l'année dernière le Prof. Hayez et Mr. Bart de Wever ; le premier avait, par voie de presse, qualifié le second d' «enfant-roi », se défendant ensuite expressément, face aux réactions virulentes, d'avoir posé là un véritable diagnostic. La déontologie des chercheurs en philosophie/psychologie politique leur interdiraitelle donc de commenter l'actualité, de dire ce qui est? Une telle attitude ne pose sans doute pas trop de problème au niveau belge, mais est vivement contestée dans des endroits plus conflictuels du globe, comme en Israël où le «silence des psychologues » (selon la formule de Daniel Bar-On) devient intenable. Se taire ou dire ce qui est, quel serait votre choix Prof. Rosoux ?

Valérie Rosoux : «Dire ce qui est »? L'objectif me paraît bien ambitieux ! En tant que philosophe et politologue, je ne choisirais pas personnellement pareille finalité. A fortiori quand il s'agit de se prononcer sur un cas qui est «le nôtre »- je veux dire, dans lequel nous sommes ancrés, que nous le voulions ou non. Dans mes recherches, le point de vue que j'adopte ne se veut guère normatif ou prescriptif. Il se veut davantage pragmatique. La question qui me guide n'est pas tant «qui a raison, qui a tort ? », mais plutôt «comment ça marche »? Il ne s'agit pas pour moi de dire ce qui serait vrai et faux, mais de contribuer à la compréhension d'un contexte. «D'où parlent les acteurs ? Quels sont leurs arguments? En quoi ces 
derniers sont-ils décalés par rapport à d'autres ? » etc. Cette posture de recherche s'est littéralement imposée à moi au fur et à mesure de mes réflexions sur les situations de post-conflit. Je vous donne un seul exemple, celui du Rwanda. La violence qui dévasta le Rwanda en 1994 est telle qu'elle semble teinter les débats scientifiques qui la concernent. Que ce soit dans la région des Grands Lacs, aux États-Unis ou en Europe, les analystes se positionnent souvent comme autant de combattants sur un champ de bataille. Cette posture de combat ne me paraît pourtant pas inévitable. L'enjeu peut être tout autre. Il s'agit pour moi de concevoir une recherche dont l'objectif n'est ni de défendre, ni de dénoncer. L'idée est de dépasser les logiques partisanes pour contribuer à la compréhension aussi fine que possible des phénomènes étudiés.

Il est utile de rappeler une fois encore que l'objet des recherches n'est pas ici uniquement l'histoire mais aussi la mémoire. La frontière qui sépare les deux notions est certes relative et poreuse. Mais il est essentiel de garder à l'esprit que le but de la mémoire ne réside pas dans la connaissance et l'intelligibilité du passé en tant que tel, mais bien dans la construction et le renforcement d'une identité. Sachant cela, quel peut être le sens de la recherche? Je serais tentée de citer Jankélévitch, qui souligne qu'il ne s'agit pas d'être «sublime », mais qu'il suffit d'être «fidèle et sérieux ». Dans cette perspective, travailler sur des zones postconflit signifie qu'il importe d'être attentif à la voix de ceux qui ne pourront plus jamais prendre la parole puisqu'elle leur a été définitivement ôtée. Mais nous nous éloignons ici beaucoup de la Belgique.

Stéphane Baele: Pour terminer cet échange, basculons notre regard, jusqu'ici focalisé sur les responsables politiques, sur l'électorat. Même si ce n'est pas l'opinion de V. Laborderie dans ce volume («Changer de système - changer de pays »), les opinions de ce dernier semblent néanmoins bien différentes au Nord et au Sud, doublant la division communautaire d'un clivage socio-économique gauchedroite. Est-il possible d'interpréter cette polarisation, ce comportement des électeurs, selon vos grilles de lecture du monde politique, Prof. Zayan?

René Zayan: D'un point de vue économique cette distinction est exacte, même si comme beaucoup de gens je ne suis effectivement pas sûr de la pertinence de la division entre la droite et la gauche. Tout d'abord parce qu'aujourd'hui tout le monde est au centre-droit ou au centre-gauche et ensuite parce que, historiquement, il y a une confusion sur l'extrême-droite et l'extrême-gauche. Il ne faut pas oublier que le fascisme, au départ, c'est en un sens l'extrême-gauche: lutte contre le capitalisme, lutte contre l'argent, lutte contre la bourgeoisie. Donc, cette distinction gauche-droite est en réalité complexe.

Ceci dit, la distinction mentionnée ici existe. En Wallonie, une communauté très travailleuse s'est appauvrie, non pas par elle-même, mais par la force des choses, par 
le fait que la Wallonie s'est déstructurée avec la crise du pétrole, le déclin de la sidérurgie. De l'autre côté, les Flamands, gagnant leur autonomie, ayant une vocation beaucoup plus grande à être anti-État belge (la Belgique étant considérée comme un symbole d'imposture), recentrent leurs intérêts sur des objectifs pragmatiques: ils travaillent pour leur argent, pour leur famille, pour leur patrimoine. En un mot, ils privilégient le travail indépendant. Il n'y a pas là une vocation à l'industrialisation, comme l'étaient les grands barons de l'industrie wallonne. De là, on peut comprendre les scores électoraux. Au Nord, le CD\&V et l'Open-VLD valorisent ainsi le travail et la famille, et d'autre part prennent en détestation le FISC. Cette mentalité, différente de celle du Sud, permet d'expliquer le succès de quelqu'un comme Jean-Marie De Decker, par exemple. A la question de savoir s'il était contre les Wallons ou contre les pauvres, il avait répondu être contre le «profitariat», sous-entendant qu'il était à la fois contre les «assistés » wallons et contre les capitaines d'industrie ou de la finance, qui n'ont jamais fait que gagner plein d'argent pour eux via l'État en amenant leurs entreprises à la faillite deux types de «profiteurs » en somme. C'est une phrase géniale que les Flamands comprennent.

Le clivage, donc, est là. On peut dire que le Vlaams Belang, la Lijst De Decker, et la N-VA, qui recueillent effectivement ensemble bon nombre de suffrages, sont tous trois des partis libéraux, de droite. À l'instar de Jean-Marie Le Pen, qui est « économiquement de droite, socialement de gauche, et, à propos de l'Europe, Français ». On peut donc avoir des partis qui sont à la fois populistes, ancrées dans certaines valeurs socialistes, et qui économiquement sont de droite.

Stéphane Baele : Je vous remercie vivement tous les deux pour cet échange ouvert, qui je l'espère s'avèrera enrichissant pour nos lecteurs, qu'ils soient de Belgique ou d'ailleurs. 